\title{
On unconditionally saturated Banach spaces
}

\author{
by \\ PANdelis Dodos and Jordi Lopez-Abad (Paris)
}

\begin{abstract}
We prove a structural property of the class of unconditionally saturated separable Banach spaces. We show, in particular, that for every analytic set $\mathcal{A}$, in the Effros-Borel space of subspaces of $C[0,1]$, of unconditionally saturated separable Banach spaces, there exists an unconditionally saturated Banach space $Y$, with a Schauder basis, that contains isomorphic copies of every space $X$ in the class $\mathcal{A}$.
\end{abstract}

\section{Introduction}

(A) An infinite-dimensional Banach space $X$ is said to be unconditionally saturated if every infinite-dimensional subspace $Y$ of $X$ contains an unconditional basic sequence. Although by the discovery of W. T. Gowers and B. Maurey $[\mathrm{GM}]$ not every separable Banach space is unconditionally saturated, this class of spaces is quite extensive, includes the "classical" ones and has some desirable closure properties (it is closed, for instance, under taking subspaces and finite sums). Most important is the fact that within the class of unconditionally saturated spaces one can develop a strong structural theory. Among the numerous results found in the literature, there are two fundamental ones that deserve special attention. The first is due to R. C. James [Ja1] and asserts that any unconditionally saturated space contains either a reflexive subspace, or $\ell_{1}$, or $c_{0}$. The second is due to A. Pełczyński $[\mathrm{P}]$ and provides a space $U$ with an unconditional basis $\left(u_{n}\right)$ with the property that any other unconditional basic sequence $\left(x_{n}\right)$, in some Banach space $X$, is equivalent to a subsequence of $\left(u_{n}\right)$.

(B) The main goal of this paper is to exhibit yet another structural property of the class of unconditionally saturated spaces which is of a global nature. To describe this property we need first to recall some standard facts. Quite often one needs a convenient way to treat separable Banach spaces as

2000 Mathematics Subject Classification: Primary 46B03, 46B15; Secondary 03E15, 46B07.

Key words and phrases: unconditional basic sequence, strongly bounded classes, $\mathcal{L}_{\infty^{-}}$ spaces. 
a unity. Such a way has been proposed by B. Bossard [Bos] and has proved to be extremely useful. More precisely, let us denote by $F(C[0,1])$ the set of all closed subspaces of the space $C[0,1]$ and let us consider the set $\mathrm{SB}=\{X \in F(C[0,1]): X$ is a linear subspace $\}$.

It is easy to see that the set SB equipped with the relative Effros-Borel structure becomes a standard Borel space (see [Bos] for more details). As $C[0,1]$ is isometrically universal for all separable Banach spaces, we may identify any class of separable Banach spaces with a subset of SB. From this point of view, we denote by US the subset of SB consisting of all $X \in \mathrm{SB}$ which are unconditionally saturated.

The above identification is ultimately related to universality problems in Banach space theory (see $[\mathrm{AD}],[\mathrm{DF}],[\mathrm{D}])$. The connection is crystalized in the following definition, introduced in [AD].

Definition 1. A class $\mathcal{C} \subseteq \mathrm{SB}$ is said to be strongly bounded if for every analytic subset $\mathcal{A}$ of $\mathcal{C}$ there exists $Y \in \mathcal{C}$ that contains isomorphic copies of every $X \in \mathcal{A}$.

In $[\mathrm{AD}$, Theorem 91(5)] it was shown that the class of unconditionally saturated Banach spaces with a Schauder basis is strongly bounded. We remove the assumption of the existence of a basis and we show the following.

TheOREM 2. Let $\mathcal{A}$ be an analytic subset of US. Then there exists an unconditionally saturated Banach space $Y$, with a Schauder basis, that contains isomorphic copies of every $X \in \mathcal{A}$. In particular, the class US is strongly bounded.

We point out that the above result is optimal. Indeed, it follows from a classical construction of J. Bourgain [Bou1] that there exists a co-analytic subset $\mathcal{B}$ of $\mathrm{SB}$ consisting of reflexive and unconditionally saturated separable Banach spaces with the following property. If $Y$ is a separable space that contains an isomorphic copy of every $X \in \mathcal{B}$, then $Y$ must contain every separable Banach space. In particular, there is no unconditionally saturated separable Banach space containing isomorphic copies of every $X \in \mathcal{B}$.

(C) By the results in $[\mathrm{AD}]$, the proof of Theorem 2 is essentially reduced to an embedding problem. Namely, given an unconditionally saturated separable Banach space $X$ one is looking for an unconditionally saturated space $Y(X)$, with a Schauder basis, that contains an isomorphic copy of $X$. In fact, for the proof of Theorem 2, one has to know additionally that this embedding is "uniform". This means, roughly, that the space $Y(X)$ is constructed from $X$ in a Borel way. In our case, the embedding problem has already been solved by J. Bourgain and G. Pisier in [BP], while its uniform version has 
recently been obtained in $[\mathrm{D}]$. These are the main ingredients of the proof of Theorem 2.

(D) At a more technical level, the paper also contains some results concerning the structure of a class of subspaces of a certain space constructed in $[\mathrm{AD}]$ and called an $\ell_{2}$ Baire sum. Specifically, we study the class of $X$-singular subspaces of an $\ell_{2}$ Baire sum and we show the following (see $\S 3.1$ for the relevant definitions):

(1) Every $X$-singular subspace is unconditionally saturated (Theorem 11 in the main text).

(2) Every $X$-singular subspace contains an $X$-compact subspace (Corollary 16). This answers a question from [AD] (see [AD, Remark 3]).

(3) Every normalized basic sequence in an $X$-singular subspace has a normalized block subsequence satisfying an upper $\ell_{2}$ estimate (Theorem 12). Hence, an $X$-singular subspace can contain no $\ell_{p}$ for $1 \leq$ $p<2$. This generalizes the fact that the 2-stopping time Banach space (see $[\mathrm{BO}]$ ) contains no $\ell_{p}$ for $1 \leq p<2$.

1.1. General notation and terminology. We shall denote by $\mathbb{N}=$ $\{0,1,2, \ldots\}$ the natural numbers. For every infinite subset $L$ of $\mathbb{N}$, we denote by $[L]$ the set of all infinite subsets of $L$. Our Banach-space-theoretic notation and terminology is standard and follows [LT], while our descriptiveset-theoretic terminology follows [Ke]. If $X$ and $Y$ are Banach spaces, then we shall denote by $X \cong Y$ the fact that $X$ and $Y$ are isomorphic.

For the convenience of the reader, let us recall the following notions. A measurable space $(X, S)$ is said to be a standard Borel space if there exists a Polish topology $\left(^{1}\right) \tau$ on $X$ such that the Borel $\sigma$-algebra of $(X, \tau)$ coincides with $S$. A subset $B$ of a standard Borel space $(X, S)$ is said to be analytic if there exists a Borel map $f: \mathbb{N}^{\mathbb{N}} \rightarrow X$ such that $f\left(\mathbb{N}^{\mathbb{N}}\right)=B$. Finally, a seminormalized sequence $\left(x_{n}\right)$ in a Banach space $X$ is said to be unconditional if there exists a constant $C>0$ such that for every $k \in \mathbb{N}$, every $F \subseteq\{0, \ldots, k\}$ and every $a_{0}, \ldots, a_{k} \in \mathbb{R}$ we have

$$
\left\|\sum_{n \in F} a_{n} x_{n}\right\| \leq C\left\|\sum_{n=0}^{k} a_{n} x_{n}\right\| .
$$

1.2. Trees. The concept of a tree has proved to be a very fruitful tool in the geometry of Banach spaces. It is also decisive throughout this work. Below we gather all the conventions concerning trees that we need.

$\left({ }^{1}\right)$ A topology $\tau$ on a set $X$ is said to be Polish if the space $(X, \tau)$ is separable and completely metrizable. 
Let $\Lambda$ be a non-empty set. We shall denote by $\Lambda^{<\mathbb{N}}$ the set of all nonempty $\left({ }^{2}\right)$ finite sequences in $\Lambda$. By $\sqsubset$ we shall denote the (strict) partial order on $\Lambda^{<\mathbb{N}}$ of end-extension. For every $\sigma \in \Lambda^{\mathbb{N}}$ and every $n \in \mathbb{N}$ with $n \geq 1$ we set $\sigma \mid n=(\sigma(0), \ldots, \sigma(n-1)) \in \Lambda^{<\mathbb{N}}$. Two nodes $s, t \in \Lambda^{<\mathbb{N}}$ are said to be comparable if either $s \sqsubseteq t$ or $t \sqsubseteq s$; otherwise they are said to be incomparable. A subset of $\Lambda^{<\mathbb{N}}$ consisting of pairwise comparable nodes is said to be a chain, while a subset of $\Lambda^{<\mathbb{N}}$ consisting of pairwise incomparable nodes is said to be an antichain.

A tree $T$ on $\Lambda$ is a subset of $\Lambda^{<\mathbb{N}}$ satisfying

$$
\forall s, t \in \Lambda^{<\mathbb{N}}(t \in T \text { and } s \sqsubset t \Rightarrow s \in T) .
$$

A tree $T$ is said to be pruned if for every $s \in T$ there exists $t \in T$ with $s \sqsubset t$. The body $[T]$ of a tree $T$ on $\Lambda$ is defined to be the set $\left\{\sigma \in \Lambda^{\mathbb{N}}\right.$ : $\sigma \mid n \in T \forall n \geq 1\}$. Notice that if $T$ is pruned, then $[T] \neq \emptyset$. A segment $\mathfrak{s}$ of a tree $T$ is a chain of $T$ satisfying

$$
\forall s, t, w \in \Lambda^{<\mathbb{N}}(s \sqsubseteq w \sqsubseteq t \text { and } s, t \in \mathfrak{s} \Rightarrow w \in \mathfrak{s}) .
$$

If $\mathfrak{s}$ is a segment of $T$, then we denote by $\min (\mathfrak{s})$ the $\sqsubseteq$-minimum node $t \in \mathfrak{s}$. We say that two segments $\mathfrak{s}$ and $\mathfrak{s}^{\prime}$ of $T$ are incomparable if for every $t \in \mathfrak{s}$ and every $t^{\prime} \in \mathfrak{s}^{\prime}$ the nodes $t$ and $t^{\prime}$ are incomparable (notice that this is equivalent to saying that $\min (\mathfrak{s})$ and $\min \left(\mathfrak{s}^{\prime}\right)$ are incomparable).

\section{Embedding unconditionally saturated spaces into spaces} with a basis. The aim of this section is to give the proof of the following result.

Proposition 3. Let $\mathcal{A}$ be an analytic subset of US. Then there exists an analytic subset $\mathcal{A}^{\prime}$ of US with the following properties:

(i) For every $Y \in \mathcal{A}^{\prime}$ the space $Y$ has a Schauder basis $\left({ }^{3}\right)$.

(ii) For every $X \in \mathcal{A}$ there exists $Y \in \mathcal{A}^{\prime}$ that contains an isometric copy of $X$.

As already mentioned in the introduction, the proof of Proposition 3 is based on a construction of $\mathcal{L}_{\infty}$-spaces due to J. Bourgain and G. Pisier [BP], as well as on its parameterized version which has recently been obtained in [D].

Let us recall, first, some definitions. If $X$ and $Y$ are two isomorphic Banach spaces (not necessarily infinite-dimensional), then their Banach-Mazur distance is defined by

$\left({ }^{2}\right)$ We should point out that in many standard textbooks, as for instance in [Ke], the empty sequence is included in $\Lambda^{<\mathbb{N}}$. We do not include it for technical reasons that will become transparent in $\S 3$.

$\left({ }^{3}\right)$ Throughout the paper, when we say that a Banach space $X$ has a Schauder basis, then we implicitly assume that $X$ is infinite-dimensional. 


$$
d(X, Y)=\inf \left\{\|T\| \cdot\left\|T^{-1}\right\|: T: X \rightarrow Y \text { is an isomorphism }\right\} .
$$

Let now $X$ be an infinite-dimensional Banach space and $\Lambda \geq 1$. The space $X$ is said to be an $\mathcal{L}_{\infty, \Lambda^{-}}$space if for every finite-dimensional subspace $F$ of $X$ there exists a finite-dimensional subspace $G$ of $X$ with $F \subseteq G$ and $d\left(G, \ell_{\infty}^{n}\right) \leq \Lambda$, where $n=\operatorname{dim}(G)$. The space $X$ is said to be an $\mathcal{L}_{\infty, \Lambda+^{-}}$ space if it is an $\mathcal{L}_{\infty, \theta}$-space for every $\theta>\Lambda$. Finally, $X$ is said to be an $\mathcal{L}_{\infty}$-space if it is $\mathcal{L}_{\infty, \Lambda}$ for some $\Lambda \geq 1$. The class of $\mathcal{L}_{\infty}$-spaces was defined by J. Lindenstrauss and A. Pełczyński [LP]. For a comprehensive account of the theory of $\mathcal{L}_{\infty}$-spaces, as well as for a presentation of many remarkable examples, we refer to the monograph of J. Bourgain [Bou2].

Let us also recall that a Banach space $X$ is said to have the Schur property if every weakly convergent sequence in $X$ is automatically norm convergent. It is an immediate consequence of Rosenthal's dichotomy [Ro] that every space $X$ with the Schur property is hereditarily $\ell_{1}$; that is, every subspace $Y$ of $X$ has a further subspace isomorphic to $\ell_{1}$ (hence, every space with the Schur property is unconditionally saturated).

The following theorem summarizes some of the basic properties of the Bourgain-Pisier construction.

Theorem 4 ([BP, Theorem 2.1]). Let $\Lambda>1$ and $X$ be a separable $B a$ nach space. Then there exists a separable $\mathcal{L}_{\infty, \Lambda+}$-space, denoted by $\mathcal{L}_{\Lambda}[X]$, which contains $X$ isometrically and is such that the quotient $\mathcal{L}_{\Lambda}[X] / X$ has the Radon-Nikodym and the Schur properties.

The parameterized version of Theorem 4 reads as follows.

Theorem 5 ([D, Theorem 16]). For every $\Lambda>1$, the set $\mathcal{L}_{\Lambda} \subseteq \mathrm{SB} \times \mathrm{SB}$ defined by

$$
(X, Y) \in \mathcal{L}_{\Lambda} \Leftrightarrow Y \text { is isometric to } \mathcal{L}_{\Lambda}[X]
$$

is analytic.

We will also need the following Ramsey-type lemma. Although it is wellknown, we sketch its proof for completeness.

Lemma 6. Let $X$ be a Banach space and $Y$ be a closed subspace of $X$. Then for every subspace $Z$ of $X$ there exists a further subspace $Z^{\prime}$ of $Z$ such that $Z^{\prime}$ is either isomorphic to a subspace of $Y$, or isomorphic to a subspace of $X / Y$. In particular, if $Y$ and $X / Y$ are both unconditionally saturated, then so is $X$.

Proof. Let $Q: X \rightarrow X / Y$ be the natural quotient map.

CASE 1: The operator $Q: Z \rightarrow X / Y$ is not strictly singular. Then, by definition, there exists a subspace $Z^{\prime}$ of $Z$ such that $\left.Q\right|_{Z^{\prime}}$ is an isomorphic embedding. 
CASE 2: The operator $Q: Z \rightarrow X / Y$ is strictly singular. In this case our hypothesis implies that for every subspace $Z^{\prime}$ of $Z$ and every $\varepsilon>0$ we may find a normalized vector $z \in Z^{\prime}$ such that $\|Q(z)\| \leq \varepsilon$. Hence, for every subspace $Z^{\prime}$ of $Z$ and every $\varepsilon>0$ there exist a normalized vector $z \in Z^{\prime}$ and a vector $y \in Y$ such that $\|z-y\|<\varepsilon$. So, we may construct a normalized Schauder basic sequence $\left(z_{n}\right)$ in $Z$ with basis constant 2 and a sequence $\left(y_{n}\right)$ in $Y$ such that $\left\|z_{n}-y_{n}\right\|<1 / 8^{n}$ for every $n \in \mathbb{N}$. It follows that $\left(y_{n}\right)$ is equivalent to $\left(z_{n}\right)$ (see [LT]). Setting $Z^{\prime}=\overline{\operatorname{span}}\left\{z_{n}: n \in \mathbb{N}\right\}$, we see that $Z^{\prime}$ is isomorphic to a subspace of $Y$.

Proof of Proposition 3. Let $\mathcal{A}$ be an analytic subset of US. Let also $\mathcal{L}_{2}$ be the subset of $\mathrm{SB} \times \mathrm{SB}$ obtained by applying Theorem 5 for $\Lambda=2$. We define $\mathcal{A}^{\prime} \subseteq$ SB by the rule

$$
Y \in \mathcal{A}^{\prime} \Leftrightarrow \exists X\left[X \in \mathcal{A} \text { and }(X, Y) \in \mathcal{L}_{2}\right] .
$$

As both $\mathcal{A}$ and $\mathcal{L}_{2}$ are analytic and the class of analytic sets is closed under projections, we see that $\mathcal{A}^{\prime}$ is analytic. We claim that $\mathcal{A}^{\prime}$ is the desired set. Indeed, notice that property (ii) is an immediate consequence of Theorem 4 . To see (i), let $Y \in \mathcal{A}^{\prime}$. There exists $X \in \mathcal{A}$ such that $Y$ is isometric to $\mathcal{L}_{2}[X]$. By Theorem 4 , we know that $\mathcal{L}_{2}[X] / X$ is unconditionally saturated. Recalling that $X$ is also unconditionally saturated, by Lemma 6 , we see that $Y \in$ US. Finally, our claim that $Y$ has a Schauder basis is an immediate consequence of the fact that $Y$ is $\mathcal{L}_{\infty}$ and of a classical result due to W. B. Johnson, H. P. Rosenthal and M. Zippin [JRZ] asserting that every separable $\mathcal{L}_{\infty}$-space has a Schauder basis.

\section{Schauder tree bases and $\ell_{2}$ Baire sums}

3.1. Definitions and statements of the main results. Let us begin by recalling the following notion.

Definition 7 ([AD, Definition 13]). Let $X$ be a Banach space, $\Lambda$ a countable set and $T$ a pruned tree on $\Lambda$. Let also $\left(x_{t}\right)_{t \in T}$ be a normalized sequence in $X$ indexed by the tree $T$. We say that $\mathfrak{X}=\left(X, \Lambda, T,\left(x_{t}\right)_{t \in T}\right)$ is a Schauder tree basis if the following are satisfied:

(a) $X=\overline{\operatorname{span}}\left\{x_{t}: t \in T\right\}$.

(b) For every $\sigma \in[T]$ the sequence $\left(x_{\sigma \mid n}\right)_{n \geq 1}$ is a (normalized) bi-monotone Schauder basic sequence.

Let $\mathfrak{X}=\left(X, \Lambda, T,\left(x_{t}\right)_{t \in T}\right)$ be a Schauder tree basis. For every $\sigma \in[T]$ we set

$$
X_{\sigma}=\overline{\operatorname{span}}\left\{x_{\sigma \mid n}: n \geq 1\right\} .
$$

Notice that in Definition 7 we do not assume that the subspace $X_{\sigma}$ of $X$ is 
complemented. Notice also that if $\sigma, \tau \in[T]$ with $\sigma \neq \tau$, then this does not necessarily imply that $X_{\sigma} \neq X_{\tau}$.

EXAmple 1. Let $X=c_{0}$ and $\left(e_{n}\right)$ be the standard unit vector basis of $c_{0}$. Let also $T=2^{<\mathbb{N}}$ be the Cantor tree, i.e. $T$ is the set of all non-empty finite sequences of 0 's and 1's. For every $t \in T$, denoting by $|t|$ the length of the finite sequence $t$, we define $x_{t}=e_{|t|-1}$. It is easy to see that the family $\left(X, 2, T,\left(x_{t}\right)_{t \in T}\right)$ is a Schauder tree basis. Observe that for every $\sigma \in[T]$ the sequence $\left(x_{\sigma \mid n}\right)_{n \geq 1}$ is the standard basis of $c_{0}$. Hence, the just defined Schauder tree basis has been obtained by "spreading" the standard basis of $c_{0}$ along the branches of $2^{<\mathbb{N}}$.

The notion of a Schauder tree basis serves as a technical vehicle for the construction of a "tree-like" Banach space in the spirit of R. C. James [Ja2]. This is the content of the following definition.

Definition 8 ([AD, §4.1]). Let $\mathfrak{X}=\left(X, \Lambda, T,\left(x_{t}\right)_{t \in T}\right)$ be a Schauder tree basis. The $\ell_{2}$ Baire sum of $\mathfrak{X}$, denoted by $T_{2}^{\mathfrak{X}}$, is defined to be the completion of $c_{00}(T)$ equipped with the norm

$$
\|z\|_{T_{2}^{\mathfrak{x}}}=\sup \left\{\left(\sum_{j=0}^{l}\left\|\sum_{t \in \mathfrak{s}_{j}} z(t) x_{t}\right\|_{X}^{2}\right)^{1 / 2}\right\}
$$

where the supremum is taken over all finite families $\left(\mathfrak{s}_{j}\right)_{j=0}^{l}$ of pairwise incomparable segments of $T$.

EXAmple 2. Let $\mathfrak{X}$ be the Schauder tree basis described in Example 1 and consider the corresponding $\ell_{2}$ Baire sum $T_{2}^{\mathfrak{X}}$. Notice that if $z \in T_{2}^{\mathfrak{X}}$, then its norm is given by the formula

$$
\|z\|_{T_{2}^{x}}=\sup \left\{\left(\sum_{j=0}^{l} z\left(t_{j}\right)^{2}\right)^{1 / 2}:\left(t_{j}\right)_{j=0}^{l} \text { is an antichain of } 2^{<\mathbb{N}}\right\} .
$$

This space has been defined by H. P. Rosenthal and it is known in the literature as the 2-stopping time Banach space (see [BO]). It is usually denoted by $S_{2}$. A very interesting fact concerning the structure of $S_{2}$ is that it contains almost isometric copies of $\ell_{p}$ for every $2 \leq p<\infty$. This is due to $\mathrm{H}$. P. Rosenthal and G. Schechtman (unpublished). On the other hand, the space $S_{2}$ contains no $\ell_{p}$ for $1 \leq p<2$.

Let $\mathfrak{X}=\left(X, \Lambda, T,\left(x_{t}\right)_{t \in T}\right)$ be a Schauder tree basis and consider the corresponding $\ell_{2}$ Baire sum $T_{2}^{\mathfrak{X}}$ of $\mathfrak{X}$. Let $\left(e_{t}\right)_{t \in T}$ be the standard Hamel basis of $c_{00}(T)$. We fix a bijection $h: T \rightarrow \mathbb{N}$ such that for every pair $t, s \in T$ we have $h(t)<h(s)$ if $t \sqsubset s$. If $\left(e_{t_{n}}\right)$ is the enumeration of $\left(e_{t}\right)_{t \in T}$ according to $h$, then it is easy to verify that the sequence $\left(e_{t_{n}}\right)$ defines a normalized bi-monotone Schauder basis of $T_{2}^{\mathfrak{X}}$. 
For every $\sigma \in[T]$ consider the subspace $\mathcal{X}_{\sigma}$ of $T_{2}^{\mathfrak{X}}$ defined by

$$
\mathcal{X}_{\sigma}=\overline{\operatorname{span}}\left\{e_{\sigma \mid n}: n \geq 1\right\} \text {. }
$$

It is easily seen that the space $\mathcal{X}_{\sigma}$ is isometric to $X_{\sigma}$, and moreover, it is 1-complemented in $T_{2}^{\mathfrak{X}}$ via the natural projection $P_{\sigma}: T_{2}^{\mathfrak{X}} \rightarrow \mathcal{X}_{\sigma}$. More generally, for every segment $\mathfrak{s}$ of $T$ we set $\mathcal{X}_{\mathfrak{s}}=\overline{\operatorname{span}}\left\{e_{t}: t \in \mathfrak{s}\right\}$. Again we see that $\mathcal{X}_{\mathfrak{s}}$ is isometric to the space $\overline{\operatorname{span}}\left\{x_{t}: t \in \mathfrak{s}\right\}$ and it is 1-complemented in $T_{2}^{\mathfrak{X}}$ via the natural projection $P_{\mathfrak{s}}: T_{2}^{\mathfrak{X}} \rightarrow \mathcal{X}_{\mathfrak{s}}$.

If $x$ is a vector in $T_{2}^{\mathfrak{X}}$, then $\operatorname{supp}(x)$ will denote its support $\{t \in T$ : $x(t) \neq 0\}$. The range of $x$, denoted by range $(x)$, is defined to be the minimal interval $I$ of $\mathbb{N}$ satisfying $\operatorname{supp}(x) \subseteq\left\{t_{n}: n \in I\right\}$. We isolate, for future use, the following consequence of the enumeration $h$ of $T$.

FACT 9 . Let $\mathfrak{s}$ be a segment of $T$ and $I$ be an interval of $\mathbb{N}$. Consider the set $\mathfrak{s}^{\prime}=\mathfrak{s} \cap\left\{t_{n}: n \in I\right\}$. Then $\mathfrak{s}^{\prime}$ is also a segment of $T$.

Let now $Y$ be a subspace of $T_{2}^{\mathfrak{X}}$. Assume that there exist a subspace $Y^{\prime}$ of $Y$ and a $\sigma \in[T]$ such that the operator $P_{\sigma}: Y^{\prime} \rightarrow \mathcal{X}_{\sigma}$ is an isomorphic embedding. In such a case, the subspace $Y$ contains information about the Schauder tree basis $\mathfrak{X}=\left(X, \Lambda, T,\left(x_{t}\right)_{t \in T}\right)$. On the other hand, there are subspaces of $T_{2}^{\mathfrak{X}}$ which are "orthogonal" to every $\mathcal{X}_{\sigma}$. These subspaces are naturally distinguished into two categories, as follows.

Definition 10 ([AD, Definition 14]). Let $\mathfrak{X}=\left(X, \Lambda, T,\left(x_{t}\right)_{t \in T}\right)$ be a Schauder tree basis and let $Y$ be a subspace of $T_{2}^{\mathfrak{X}}$. We say that $Y$ is:

(a) $X$-singular if $P_{\sigma}: Y \rightarrow \mathcal{X}_{\sigma}$ is strictly singular for every $\sigma \in[T]$.

(b) $X$-compact $P_{\sigma}: Y \rightarrow \mathcal{X}_{\sigma}$ is compact if for every $\sigma \in[T]$.

In this section, we focus on the structure of the class of $X$-singular subspaces of an arbitrary $\ell_{2}$ Baire sum. Our main results are summarized below.

Theorem 11. Let $\mathfrak{X}=\left(X, \Lambda, T,\left(x_{t}\right)_{t \in T}\right)$ be a Schauder tree basis and $Y$ be an $X$-singular subspace of $T_{2}^{\mathfrak{X}}$. Then $Y$ is unconditionally saturated.

Theorem 12. Let $\mathfrak{X}=\left(X, \Lambda, T,\left(x_{t}\right)_{t \in T}\right)$ be a Schauder tree basis and $Y$ be an $X$-singular subspace of $T_{2}^{\mathfrak{X}}$. Then for every normalized Schauder basic sequence $\left(x_{n}\right)$ in $Y$ there exists a normalized block sequence $\left(y_{n}\right)$ of $\left(x_{n}\right)$ satisfying an upper $\ell_{2}$ estimate. That is, there exists a constant $C \geq 1$ such that for every $k \in \mathbb{N}$ and every $a_{0}, \ldots, a_{k} \in \mathbb{R}$ we have

$$
\left\|\sum_{n=0}^{k} a_{n} y_{n}\right\|_{T_{2}^{\mathfrak{x}}} \leq C\left(\sum_{n=0}^{k}\left|a_{n}\right|^{2}\right)^{1 / 2} .
$$

In particular, an $X$-singular subspace $Y$ of $T_{2}^{\mathfrak{X}}$ contains no $\ell_{p}$ for $1 \leq p<2$.

We notice that in Theorem 12 one cannot expect to obtain a block sequence satisfying a lower $\ell_{2}$ estimate. Indeed, as shown in [AD, Theorem 25], 
if $\mathfrak{X}=\left(X, \Lambda, T,\left(x_{t}\right)_{t \in T}\right)$ is a Schauder tree basis such that the tree $T$ is not small (precisely, if $T$ contains a perfect $\left({ }^{4}\right)$ subtree), then one can find in $T_{2}^{\mathfrak{X}}$ a normalized block sequence $\left(x_{n}\right)$ which is equivalent to the standard basis of $c_{0}$ and which spans an $X$-singular subspace. Clearly, no block subsequence of $\left(x_{n}\right)$ has a lower $\ell_{2}$ estimate.

The rest of this section is organized as follows. In $\S 3.2$ we provide a characterization of the class of $X$-singular subspaces of $T_{2}^{\mathfrak{X}}$. Using this characterization we show, for instance, that every $X$-singular subspace of $T_{2}^{\mathfrak{X}}$ contains an $X$-compact subspace. This can be seen as a "tree version" of the classical theorem of $T$. Kato asserting that for every strictly singular operator $T: X \rightarrow Y$ there is an infinite-dimensional subspace $Z$ of $X$ such that the operator $T: Z \rightarrow Y$ is compact. In $\S 3.3$ we give the proofs of Theorems 11 and 12 .

3.2. A characterization of $X$-singular subspaces. We start with the following definition.

Definition 13. Let $\mathfrak{X}=\left(X, \Lambda, T,\left(x_{t}\right)_{t \in T}\right)$ be a Schauder tree basis. The $c_{0}$ Baire sum of $\mathfrak{X}$, denoted by $T_{0}^{\mathfrak{X}}$, is defined to be the completion of $c_{00}(T)$ equipped with the norm

$$
\|z\|_{T_{0}^{\mathfrak{x}}}=\sup \left\{\left\|\sum_{t \in \mathfrak{s}} z(t) x_{t}\right\|_{X}: \mathfrak{s} \text { is a segment of } T\right\} .
$$

We shall denote by I : $T_{2}^{\mathfrak{X}} \rightarrow T_{0}^{\mathfrak{X}}$ the natural inclusion operator.

Our characterization of $X$-singular subspaces of $T_{2}^{\mathfrak{X}}$ is achieved by considering the functional-analytic properties of the inclusion operator $\mathrm{I}: T_{2}^{\mathfrak{X}} \rightarrow T_{0}^{\mathfrak{X}}$. Precisely, we have the following.

Proposition 14. Let $\mathfrak{X}=\left(X, \Lambda, T,\left(x_{t}\right)_{t \in T}\right)$ be a Schauder tree basis. Let $Y$ be a subspace of $T_{2}^{\mathfrak{X}}$. Then the following are equivalent.

(i) $Y$ is an $X$-singular subspace of $T_{2}^{\mathfrak{X}}$.

(ii) The operator $\mathrm{I}: Y \rightarrow T_{0}^{\mathfrak{X}}$ is strictly singular.

Let us isolate two consequences of Proposition 14. The first one is simply a restatement of Proposition 14.

Corollary 15. Let $\mathfrak{X}=\left(X, \Lambda, T,\left(x_{t}\right)_{t \in T}\right)$ be a Schauder tree basis and $Y$ be a block subspace of $T_{2}^{\mathfrak{X}}$. Assume that $Y$ is $X$-singular. Then for every $\varepsilon>0$ we may find a finitely supported vector $y \in Y$ with $\|y\|=1$ and such that $\left\|P_{\mathfrak{s}}(y)\right\| \leq \varepsilon$ for every segment $\mathfrak{s}$ of $T$.

Corollary 16. Let $\mathfrak{X}=\left(X, \Lambda, T,\left(x_{t}\right)_{t \in T}\right)$ be a Schauder tree basis and $Y$ be an infinite-dimensional subspace of $T_{2}^{\mathfrak{X}}$. Assume that $Y$ is $X$ -

$\left({ }^{4}\right)$ A tree $T$ is perfect if every node $t \in T$ has at least two incomparable successors. 
singular. Then there exists an infinite-dimensional subspace $Y^{\prime}$ of $Y$ which is $X$-compact.

Proof. By Proposition 14, the operator I : $Y \rightarrow T_{0}^{\mathfrak{X}}$ is strictly singular. By [LT, Proposition 2.c.4], there exists an infinite-dimensional subspace $Y^{\prime}$ of $Y$ such that the operator $\mathrm{I}: Y^{\prime} \rightarrow T_{0}^{\mathfrak{X}}$ is compact. It is easy to see that $Y^{\prime}$ must be an $X$-compact subspace of $T_{2}^{\mathfrak{X}}$ in the sense of Definition 10(b).

For the proof of Proposition 14 we need a couple of results from [AD]. The first one is the following (see [AD, Lemma 17]).

Lemma 17. Let $\left(x_{n}\right)$ be a bounded block sequence in $T_{2}^{\mathfrak{X}}$ and $\varepsilon>0$ be such that $\lim \sup \left\|P_{\sigma}\left(x_{n}\right)\right\|<\varepsilon$ for every $\sigma \in[T]$. Then there exists $L \in[\mathbb{N}]$ such that for every $\sigma \in[T]$ we have $\left|\left\{n \in L:\left\|P_{\sigma}\left(x_{n}\right)\right\| \geq \varepsilon\right\}\right| \leq 1$.

The second result is the following special case of [AD, Proposition 33].

Proposition 18. Let $Y$ be a block $X$-singular subspace of $T_{2}^{\mathfrak{X}}$. Then for every $\varepsilon>0$ we may find a normalized block sequence $\left(y_{n}\right)$ in $Y$ such that for every $\sigma \in[T]$ we have limsup $\left\|P_{\sigma}\left(y_{n}\right)\right\|<\varepsilon$.

We are ready to proceed to the proof of Proposition 14.

Proof of Proposition 14. It is clear that (ii) implies (i). Hence we only need to show the reverse implication. We argue by contradiction. So, assume that $Y$ is an $X$-singular subspace of $T_{2}^{\mathfrak{X}}$ such that the operator I : $Y \rightarrow T_{0}^{\mathfrak{X}}$ is not strictly singular. By definition, there exists a further subspace $Y^{\prime}$ of $Y$ such that $\mathrm{I}: Y^{\prime} \rightarrow T_{0}^{\mathfrak{X}}$ is an isomorphic embedding. Using a sliding hump argument, we may recursively select a normalized basic sequence $\left(y_{n}\right)$ in $Y^{\prime}$ and a normalized block sequence $\left(z_{n}\right)$ in $T_{2}^{\mathfrak{X}}$ such that, with $Z=\overline{\operatorname{span}}\left\{z_{n}\right.$ : $n \in \mathbb{N}\}$, the following are satisfied:

(a) The sequence $\left(z_{n}\right)$ is equivalent to $\left(y_{n}\right)$.

(b) The subspace $Z$ of $T_{2}^{\mathfrak{X}}$ is $X$-singular.

(c) The operator I : $Z \rightarrow T_{0}^{\mathfrak{X}}$ is an isomorphic embedding.

The selection is fairly standard (we leave the details to the interested reader). By (c) above, there exists a constant $C>0$ such that for every $z \in Z$ we have

$$
C\|z\|_{T_{2}^{\mathfrak{x}}} \leq\|z\|_{T_{0}^{\mathfrak{x}}} \leq\|z\|_{T_{2}^{\mathfrak{x}}} .
$$

We fix $k_{0} \in \mathbb{N}$ and $\varepsilon>0$ satisfying

$$
k_{0}>64 / C^{4} \text { and } \varepsilon<\min \left\{C / 2,1 / k_{0}\right\} .
$$

By (b) above, we may apply Proposition 18 to the block subspace $Z$ of $T_{2}^{\mathfrak{X}}$ and the chosen $\varepsilon$. It follows that there exists a normalized block sequence $\left(x_{n}\right)$ in $Z$ such that $\limsup \left\|P_{\sigma}\left(x_{n}\right)\right\|<\varepsilon$ for every $\sigma \in[T]$. By Lemma 17 and by passing to a subsequence of $\left(x_{n}\right)$ if necessary, we may additionally 
assume that for every $\sigma \in[T]$ we have $\left|\left\{n \in \mathbb{N}:|| P_{\sigma}\left(x_{n}\right) \| \geq \varepsilon\right\}\right| \leq 1$. As the basis of $T_{2}^{\mathfrak{X}}$ is bi-monotone, we may strengthen this property to the following one:

(d) For every segment $\mathfrak{s}$ of $T$ we have $\left|\left\{n \in \mathbb{N}:\left\|P_{\mathfrak{s}}\left(x_{n}\right)\right\| \geq \varepsilon\right\}\right| \leq 1$.

By Fact 9 and (10), for every $n \in \mathbb{N}$ we may select a segment $\mathfrak{s}_{n}$ of $T$ such that:

(e) $\left\|P_{\mathfrak{s}_{n}}\left(x_{n}\right)\right\| \geq C$.

(f) $\mathfrak{s}_{n} \subseteq\left\{t_{k}: k \in \operatorname{range}\left(x_{n}\right)\right\}$.

As $\left(x_{n}\right)$ is a block sequence, we see that such a selection guarantees that

(g) $\left\|P_{\mathfrak{s}_{n}}\left(x_{m}\right)\right\|=0$ for all $n, m \in \mathbb{N}$ with $n \neq m$.

We set $t_{n}=\min \left(\mathfrak{s}_{n}\right)$. Applying the classical Ramsey theorem we find an infinite subset $L=\left\{l_{0}<l_{1}<\cdots\right\}$ of $\mathbb{N}$ such that one of the following (mutually exclusive) cases must occur.

CASE 1: The set $\left\{t_{n}: n \in L\right\}$ is an antichain. In this case our hypothesis implies that for every $n, m \in L$ with $n \neq m$ the segments $\mathfrak{s}_{n}$ and $\mathfrak{s}_{m}$ are incomparable. We define $z=x_{l_{0}}+\cdots+x_{l_{k_{0}}}$. As the family $\left(\mathfrak{s}_{l_{i}}\right)_{i=0}^{k_{0}}$ consists of pairwise incomparable segments of $T$, we get

$$
\|z\| \geq\left(\sum_{i=0}^{k_{0}}\left\|P_{\mathfrak{s}_{i}}(z)\right\|^{2}\right)^{1 / 2} \stackrel{(\mathrm{g})}{=}\left(\sum_{i=0}^{k_{0}}\left\|P_{\mathfrak{s}_{l_{i}}}\left(x_{l_{i}}\right)\right\|^{2}\right)^{1 / 2} \stackrel{(\mathrm{e})}{\geq} C \sqrt{k_{0}+1} .
$$

Now we set $w=z /\|z\| \in Z$. Invoking (d) above, inequality (12) and the choice of $k_{0}$ and $\varepsilon$ made in (11), for every segment $\mathfrak{s}$ of $T$ we have

$$
\left\|P_{\mathfrak{s}}(w)\right\| \leq \frac{1+k_{0} \varepsilon}{C \sqrt{k_{0}+1}}<\frac{C}{2} .
$$

It follows that

$$
\|w\|_{T_{0}^{\mathfrak{x}}} \leq C / 2,
$$

which contradicts inequality (10). Hence this case is impossible.

CASE 2: The set $\left\{t_{n}: n \in L\right\}$ is a chain. Let $\tau \in[T]$ be the branch of $T$ determined by the infinite chain $\left\{t_{n}: n \in L\right\}$. By (d) above and by passing to an infinite subset of $L$ if necessary, we may assume that $\left\|P_{\tau}\left(x_{n}\right)\right\|<\varepsilon$ for every $n \in L$. The basis of $T_{2}^{\mathfrak{X}}$ is bi-monotone, and so we have the following property.

(h) If $\mathfrak{s}$ is a segment of $T$ with $\mathfrak{s} \subseteq \tau$, then $\left\|P_{\mathfrak{s}}\left(x_{n}\right)\right\|<\varepsilon$ for every $n \in L$. We set $\mathfrak{s}_{n}^{\prime}=\mathfrak{s}_{n} \backslash \tau$. Observe that $\mathfrak{s}_{n}^{\prime}$ is a subsegment of $\mathfrak{s}_{n}$. Notice that $\mathfrak{s}_{n}$ is the disjoint union of the successive segments $\mathfrak{s}_{n} \cap \tau$ and $\mathfrak{s}_{n}^{\prime}$. Hence, by properties (e) and (h) above and the choice of $\varepsilon$, we see that

$$
\left\|P_{\mathfrak{s}_{n}^{\prime}}\left(x_{n}\right)\right\| \geq C-\varepsilon \geq C / 2
$$


for every $n \in L$. Notice also that if $n, m \in L$ with $n \neq m$, then the segments $\mathfrak{s}_{n}^{\prime}$ and $\mathfrak{s}_{m}^{\prime}$ are incomparable. We set

$$
z=x_{l_{0}}+\cdots+x_{l_{k_{0}}} \text { and } w=z /\|z\| .
$$

Arguing precisely as in Case 1 and using the estimate in (13), we conclude that

$$
\|w\|_{T_{0}^{\mathfrak{x}}} \leq C / 2 .
$$

This is again a contradiction.

3.3. Proof of Theorems 11 and 12. We start with the following lemma.

Lemma 19. Let $\mathfrak{X}=\left(X, \Lambda, T,\left(x_{t}\right)_{t \in T}\right)$ be a Schauder tree basis. Let $\left(w_{n}\right)$ be a normalized block sequence in $T_{2}^{\mathfrak{X}}$ such that for every $n \in \mathbb{N}$ with $n \geq 1$ and every segment $\mathfrak{s}$ of $T$ we have

$$
\left\|P_{\mathfrak{s}}\left(w_{n}\right)\right\| \leq \frac{1}{\sum_{i=0}^{n-1}\left|\operatorname{supp}\left(w_{i}\right)\right|^{1 / 2}} \cdot \frac{1}{2^{n+2}} .
$$

Then the following are satisfied.

(i) The sequence $\left(w_{n}\right)$ is unconditional.

(ii) The sequence $\left(w_{n}\right)$ satisfies an upper $\ell_{2}$ estimate.

Proof. We will only give the proof of (i). For a proof of (ii) we refer to [AD, Proposition 21].

So, let $k \in \mathbb{N}$ and $a_{0}, \ldots, a_{k} \in \mathbb{R}$ be such that $\left\|\sum_{n=0}^{k} a_{n} w_{n}\right\|=1$. Let also $F \subseteq\{0, \ldots, k\}$ with $F=\left\{n_{0}<\cdots<n_{p}\right\}$ its increasing enumeration. We will show that $\left\|\sum_{n \in F} a_{n} w_{n}\right\| \leq \sqrt{3}$. This will clearly finish the proof. For notational simplicity, we set

$$
w=\sum_{n=0}^{k} a_{n} w_{n} \quad \text { and } \quad z=\sum_{n \in F} a_{n} w_{n} .
$$

Let $\left(\mathfrak{s}_{j}\right)_{j=0}^{l}$ be an arbitrary collection of pairwise incomparable segments of $T$. We want to estimate the sum $\sum_{j=0}^{l}\left\|P_{\mathfrak{s}_{j}}(z)\right\|^{2}$. To this end, we may assume that for every $j \in\{0, \ldots, l\}$ there exists $i \in\{0, \ldots, p\}$ with $\mathfrak{s}_{j} \cap$ $\operatorname{supp}\left(w_{n_{i}}\right) \neq \emptyset$. We define recursively a partition $\left(\Delta_{i}\right)_{i=0}^{p}$ of $\{0, \ldots, l\}$ by the rule

$$
\begin{aligned}
\Delta_{0} & =\left\{j \in\{0, \ldots, l\}: \mathfrak{s}_{j} \cap \operatorname{supp}\left(w_{n_{0}}\right) \neq \emptyset\right\} \\
\Delta_{1} & =\left\{j \in\{0, \ldots, l\} \backslash \Delta_{0}: \mathfrak{s}_{j} \cap \operatorname{supp}\left(w_{n_{1}}\right) \neq \emptyset\right\}, \\
& \vdots \\
\Delta_{p} & =\left\{j \in\{0, \ldots, l\} \backslash \bigcup_{i=0}^{p-1} \Delta_{i}: \mathfrak{s}_{j} \cap \operatorname{supp}\left(w_{n_{p}}\right) \neq \emptyset\right\} .
\end{aligned}
$$


The segments $\left(\mathfrak{s}_{j}\right)_{j=0}^{l}$ are pairwise incomparable and a fortiori disjoint. It follows that

$$
\left|\Delta_{i}\right| \leq\left|\operatorname{supp}\left(w_{n_{i}}\right)\right| \quad \text { for every } i \in\{0, \ldots, p\} .
$$

Notice also that for every $0 \leq i<q \leq p$ we have

$$
\sum_{j \in \Delta_{q}}\left\|P_{\mathfrak{s}_{j}}\left(w_{n_{i}}\right)\right\|=0 .
$$

Let $j \in\{0, \ldots, l\}$. There exists a unique $i \in\{0, \ldots, p\}$ such that $j \in \Delta_{i}$. By Fact 9 , we may select a segment $\mathfrak{s}_{j}^{\prime}$ of $T$ such that:

(a) $\mathfrak{s}_{j}^{\prime} \subseteq \mathfrak{s}_{j}$

(b) $\mathfrak{s}_{j}^{\prime} \subseteq\left\{t_{m}: m \in\right.$ range $\left.\left(w_{n_{i}}\right)\right\}$.

(c) $\left\|P_{\mathfrak{s}_{j}}\left(a_{n_{i}} w_{n_{i}}\right)\right\|=\left\|P_{\mathfrak{s}_{j}^{\prime}}\left(a_{n_{i}} w_{n_{i}}\right)\right\|$.

The above selection guarantees the following properties:

(d) The family $\left(\mathfrak{s}_{j}^{\prime}\right)_{j=0}^{l}$ consists of pairwise incomparable segments of $T$. This is a straightforward consequence of (a) above and of our assumptions on the family $\left(\mathfrak{s}_{j}\right)_{j=0}^{l}$.

(e) We have $\left\|P_{\mathfrak{s}_{j}}\left(a_{n_{i}} w_{n_{i}}\right)\right\|=\left\|P_{\mathfrak{s}_{j}^{\prime}}\left(a_{n_{i}} w_{n_{i}}\right)\right\|=\left\|P_{\mathfrak{s}_{j}^{\prime}}(w)\right\|$. This is a consequence of (b) and (c) above and of the fact that $\left(w_{n}\right)$ is a block sequence.

We are ready for the final part of the argument. Let $i \in\{0, \ldots, p\}$ and $j \in \Delta_{i}$. Our goal is to estimate the quantity $\left\|P_{\mathfrak{s}_{j}}(z)\right\|$. First we notice that

$$
\begin{aligned}
&\left\|P_{\mathfrak{s}_{j}}(z)\right\| \stackrel{(16)}{=}\left\|P_{\mathfrak{s}_{j}}\left(a_{n_{i}} w_{n_{i}}+\cdots+a_{n_{p}} w_{n_{p}}\right)\right\| \\
& \leq\left\|P_{\mathfrak{s}_{j}}\left(a_{n_{i}} w_{n_{i}}\right)\right\|+\sum_{q=i+1}^{p}\left|a_{n_{q}}\right| \cdot\left\|P_{\mathfrak{s}_{j}}\left(w_{n_{q}}\right)\right\| .
\end{aligned}
$$

Invoking (14) and the fact that the Schauder basis $\left(e_{t}\right)_{t \in T}$ of $T_{2}^{\mathfrak{X}}$ is bimonotone, we see that for every $q \in\{i+1, \ldots, p\}$ we have $\left\|P_{\mathfrak{s}_{j}}\left(w_{n_{q}}\right)\right\| \leq$ $\left|\operatorname{supp}\left(w_{n_{i}}\right)\right|^{-1 / 2} \cdot 2^{-(q+2)}$ and $\left|a_{n_{q}}\right| \leq 1$. Hence, the previous estimate yields

$$
\begin{aligned}
\left\|P_{\mathfrak{s}_{j}}(z)\right\| & \leq\left\|P_{\mathfrak{s}_{j}}\left(a_{n_{i}} w_{n_{i}}\right)\right\|+\frac{1}{\left|\operatorname{supp}\left(w_{n_{i}}\right)\right|^{1 / 2}} \cdot \sum_{q=i+1}^{p} \frac{1}{2^{q+2}} \\
& \stackrel{(15)}{\leq}\left\|P_{\mathfrak{s}_{j}}\left(a_{n_{i}} w_{n_{i}}\right)\right\|+\frac{1}{\left|\Delta_{i}\right|^{1 / 2}} \cdot \frac{1}{2^{i+2}} \\
& \stackrel{(\mathrm{e})}{=}\left\|P_{\mathfrak{s}_{j}}(w)\right\|+\frac{1}{\left|\Delta_{i}\right|^{1 / 2}} \cdot \frac{1}{2^{i+2}} .
\end{aligned}
$$


The above inequality, in turn, implies that if $\Delta_{i}$ is non-empty, then

$$
\begin{aligned}
\sum_{j \in \Delta_{i}}\left\|P_{\mathfrak{s}_{j}}(z)\right\|^{2} & \leq 2 \sum_{j \in \Delta_{i}}\left\|P_{\mathfrak{s}_{j}^{\prime}}(w)\right\|^{2}+2 \sum_{j \in \Delta_{i}} \frac{1}{\left|\Delta_{i}\right|} \cdot \frac{1}{2^{i+2}} \\
& \leq 2 \sum_{j \in \Delta_{i}}\left\|P_{\mathfrak{s}_{j}^{\prime}}(w)\right\|^{2}+\frac{1}{2^{i+1}} .
\end{aligned}
$$

Summarizing, we see that

$\sum_{j=0}^{l}\left\|P_{\mathfrak{s}_{j}}(z)\right\|^{2}=\sum_{i=0}^{p} \sum_{j \in \Delta_{i}}\left\|P_{\mathfrak{s}_{j}}(z)\right\|^{2} \stackrel{(17)}{\leq} 2 \sum_{j=0}^{l}\left\|P_{\mathfrak{s}_{j}^{\prime}}(w)\right\|^{2}+1 \stackrel{(\mathrm{d})}{\leq} 2\|w\|^{2}+1 \leq 3$.

The family $\left(\mathfrak{s}_{j}\right)_{j=0}^{l}$ was arbitrary, and so $\|z\| \leq \sqrt{3}$.

Proof of Theorem 11. Let $Y$ be an $X$-singular subspace of $T_{2}^{\mathfrak{X}}$. Clearly, every subspace $Y^{\prime}$ of $Y$ is also $X$-singular. Hence, it is enough to show that every $X$-singular subspace contains an unconditional basic sequence. So, let $Y$ be one. Using a sliding hump argument, we may additionally assume that $Y$ is a block subspace of $T_{2}^{\mathfrak{X}}$. Recursively and with the help of Corollary 15, we may construct a normalized block sequence $\left(w_{n}\right)$ in $Y$ such that for every $n \in \mathbb{N}$ with $n \geq 1$ and every segment $\mathfrak{s}$ of $T$ we have

$$
\left\|P_{\mathfrak{s}}\left(w_{n}\right)\right\| \leq \frac{1}{\sum_{i=0}^{n-1}\left|\operatorname{supp}\left(w_{i}\right)\right|^{1 / 2}} \cdot \frac{1}{2^{n+2}} .
$$

By Lemma 19(i), the sequence $\left(w_{n}\right)$ is unconditional.

We proceed to the proof of Theorem 12 .

Proof of Theorem 12. Let $Y$ be an $X$-singular subspace of $T_{2}^{\mathfrak{X}}$. Let also $\left(x_{n}\right)$ be a normalized Schauder basic sequence in $Y$. A standard sliding hump argument allows us to construct a normalized block sequence $\left(v_{n}\right)$ of $\left(x_{n}\right)$ and a block sequence $\left(z_{n}\right)$ in $T_{2}^{\mathfrak{X}}$ such that, with $Z=\overline{\operatorname{span}}\left\{z_{n}: n \in \mathbb{N}\right\}$, the following are satisfied:

(a) The sequences $\left(v_{n}\right)$ and $\left(z_{n}\right)$ are equivalent.

(b) The subspace $Z$ of $T_{2}^{\mathfrak{X}}$ is $X$-singular.

As in the proof of Theorem 11, using (b) above and Corollary 15, we construct a normalized block sequence $\left(w_{n}\right)$ of $\left(z_{n}\right)$ such that for every $n \in \mathbb{N}$ with $n \geq 1$ and every segment $\mathfrak{s}$ of $T$ inequality (14) is satisfied for the sequence $\left(w_{n}\right)$. By Lemma 19(ii), the sequence $\left(w_{n}\right)$ satisfies an upper $\ell_{2}$ estimate. Let $\left(b_{n}\right)$ be the block sequence of $\left(v_{n}\right)$ corresponding to $\left(w_{n}\right)$. Observe that, by (a) above, $\left(b_{n}\right)$ is seminormalized and satisfies an upper $\ell_{2}$ estimate. The property of being a block sequence is transitive, and so $\left(b_{n}\right)$ is a normalized block sequence of $\left(x_{n}\right)$ as well. Hence, setting $y_{n}=b_{n} /\left\|b_{n}\right\|$ for every $n \in \mathbb{N}$, we see that the sequence $\left(y_{n}\right)$ is as desired. 
Finally, to see that an $X$-singular subspace of $T_{2}^{\mathfrak{X}}$ can contain no $\ell_{p}$ for $1 \leq p<2$, we argue by contradiction. So, assume that $Y$ is an $X$-singular subspace of $T_{2}^{\mathfrak{X}}$ containing an isomorphic copy of $\ell_{p_{0}}$ for some $1 \leq p_{0}<2$. There exists, in such a case, a normalized basic sequence $\left(x_{n}\right)$ in $Y$ which is equivalent to the standard unit vector basis $\left(e_{n}\right)$ of $\ell_{p_{0}}$. Let $\left(y_{n}\right)$ be a normalized block subsequence of $\left(x_{n}\right)$ satisfying an upper $\ell_{2}$ estimate. As any normalized block subsequence of $\left(e_{n}\right)$ is equivalent to $\left(e_{n}\right)$ (see [LT]), we see that there must exist constants $C \geq c>0$ such that for every $k \in \mathbb{N}$ and any $a_{0}, \ldots, a_{k} \in \mathbb{R}$ we have

$$
c\left(\sum_{n=0}^{k}\left|a_{n}\right|^{p_{0}}\right)^{1 / p_{0}} \leq\left\|\sum_{n=0}^{k} a_{n} y_{n}\right\|_{T_{2}^{x}} \leq C\left(\sum_{n=0}^{k}\left|a_{n}\right|^{2}\right)^{1 / 2} .
$$

This is clearly a contradiction.

We close this section by recording the following consequence of Theorem 12 .

Corollary 20. Let $\mathfrak{X}=\left(X, \Lambda, T,\left(x_{t}\right)_{t \in T}\right)$ be a Schauder tree basis. Let $1 \leq p<2$. Then the following are equivalent.

(i) The space $T_{2}^{\mathfrak{X}}$ contains an isomorphic copy of $\ell_{p}$.

(ii) There exists $\sigma \in[T]$ such that $X_{\sigma}$ contains an isomorphic copy of $\ell_{p}$.

Proof. It is clear that (ii) implies (i). Conversely, assume that $\ell_{p}$ embeds into $T_{2}^{\mathfrak{X}}$ and let $Y$ be a subspace of $T_{2}^{\mathfrak{X}}$ which is isomorphic to $\ell_{p}$. By Theorem 12, we see that $Y$ is not $X$-singular. Hence, there exist $\sigma \in[T]$ and an infinite-dimensional subspace $Y^{\prime}$ of $Y$ such that $P_{\sigma}: Y^{\prime} \rightarrow \mathcal{X}_{\sigma}$ is an isomorphic embedding. Recalling that every subspace of $\ell_{p}$ contains a copy of $\ell_{p}$ and that the spaces $\mathcal{X}_{\sigma}$ and $X_{\sigma}$ are isometric yields the result.

4. The main result. This section is devoted to the proof of Theorem 2 stated in the introduction. To this end, we will need the following correspondence principle between analytic classes of separable Banach spaces and Schauder tree bases (see [AD, Proposition 83] or [D, Lemma 32]):

Lemma 21. Let $\mathcal{A}^{\prime}$ be an analytic subset of SB such that every $Y \in \mathcal{A}^{\prime}$ has a Schauder basis. Then there exist a separable Banach space $X$, a pruned tree $T$ on $\mathbb{N} \times \mathbb{N}$ and a normalized sequence $\left(x_{t}\right)_{t \in T}$ in $X$ such that the following are satisfied.

(i) The family $\mathfrak{X}=\left(X, \Lambda, T,\left(x_{t}\right)_{t \in T}\right)$ is a Schauder tree basis.

(ii) For every $Y \in \mathcal{A}^{\prime}$ there exists $\sigma \in[T]$ with $Y \cong X_{\sigma}$.

(iii) For every $\sigma \in[T]$ there exists $Y \in \mathcal{A}^{\prime}$ with $X_{\sigma} \cong Y$.

Proof of Theorem 2. Let $\mathcal{A}$ be an analytic subset of US. We apply Proposition 3 and we get a subset $\mathcal{A}^{\prime}$ of SB with the following properties: 
(a) The set $\mathcal{A}^{\prime}$ is analytic.

(b) Every $Y \in \mathcal{A}^{\prime}$ has a Schauder basis.

(c) Every $Y \in \mathcal{A}^{\prime}$ is unconditionally saturated.

(d) For every $X \in \mathcal{A}$ there exists $Y \in \mathcal{A}^{\prime}$ such that $Y$ contains an isometric copy of $X$.

By (a) and (b) above, we can apply Lemma 21 to the set $\mathcal{A}^{\prime}$ to get a Schauder tree basis $\mathfrak{X}=\left(X, \Lambda, T,\left(x_{t}\right)_{t \in T}\right)$ satisfying the following.

(e) For every $Y \in \mathcal{A}^{\prime}$ there exists $\sigma \in[T]$ with $Y \cong X_{\sigma}$.

(f) For every $\sigma \in[T]$ there exists $Y \in \mathcal{A}^{\prime}$ such that $X_{\sigma} \cong Y$.

Consider the $\ell_{2}$ Baire sum $T_{2}^{\mathfrak{X}}$ of this Schauder tree basis $\mathfrak{X}$. We claim that the space $T_{2}^{\mathfrak{X}}$ is as desired. Indeed, recall first that $T_{2}^{\mathfrak{X}}$ has a Schauder basis. Moreover, by (d) and (e) above we see that $T_{2}^{\mathfrak{X}}$ contains an isomorphic copy of every $X \in \mathcal{A}$.

What remains is to check that $T_{2}^{\mathfrak{X}}$ is unconditionally saturated. To this end, let $Z$ be an arbitrary subspace of $T_{2}^{\mathfrak{X}}$. We have to show that $Z$ contains an unconditional basic sequence.

CASE 1: The subspace $Z$ is not $X$-singular. In this case, by definition, there exist $\sigma \in[T]$ and a further subspace $Z^{\prime}$ of $Z$ such that the operator $P_{\sigma}: Z^{\prime} \rightarrow \mathcal{X}_{\sigma}$ is an isomorphic embedding. By (f) and (c) above, $Z^{\prime}$ must contain an unconditional basic sequence.

CASE 2: The subspace $Z$ is $X$-singular. By Theorem 11, in this case $Z$ must also contain an unconditional basic sequence.

By the above, $T_{2}^{\mathfrak{X}}$ is unconditionally saturated.

\section{References}

[AD] S. A. Argyros and P. Dodos, Genericity and amalgamation of classes of Banach spaces, Adv. Math. 209 (2007), 666-748.

[BO] H. Bang and E. Odell, On the stopping time Banach space, Quart. J. Math. Oxford 40 (1989), 257-273.

[Bos] B. Bossard, A coding of separable Banach spaces. Analytic and co-analytic families of Banach spaces, Fund. Math. 172 (2002), 117-152.

[Bou1] J. Bourgain, On separable Banach spaces, universal for all separable reflexive spaces, Proc. Amer. Math. Soc. 79 (1980), 241-246.

[Bou2] - New Classes of $\mathcal{L}^{p}$-Spaces, Lecture Notes in Math. 889, Springer, 1981.

[BP] J. Bourgain and G. Pisier, A construction of $\mathcal{L}_{\infty}$-spaces and related Banach spaces, Bol. Soc. Brasil. Mat. 14 (1983), 109-123.

[D] P. Dodos, On classes of Banach spaces admitting "small" universal spaces, Trans. Amer. Math. Soc., to appear.

[DF] P. Dodos and V. Ferenczi, Some strongly bounded classes of Banach spaces, Fund. Math. 193 (2007), 171-179. 
[GM] W. T. Gowers and B. Maurey, The unconditional basic sequence problem, J. Amer. Math. Soc. 6 (1993), 851-874.

[Ja1] R. C. James, Bases and reflexivity of Banach spaces, Ann. of Math. 52 (1950), $518-527$.

[Ja2] —, A separable somewhat reflexive Banach space with non-separable dual, Bull. Amer. Math. Soc. 80 (1974), 738-743.

[JRZ] W. B. Johnson, H. P. Rosenthal and M. Zippin, On bases, finite-dimensional decompositions and weaker structures in Banach spaces, Israel J. Math. 9 (1971), 488-506.

[Ke] A. S. Kechris, Classical Descriptive Set Theory, Grad. Texts in Math. 156, Springer, 1995.

[LP] J. Lindenstrauss and A. Pełczyński, Absolutely summing operators in $\mathcal{L}_{p}$ spaces and their applications, Studia Math. 29 (1968), 275-326.

[LT] J. Lindenstrauss and L. Tzafriri, Classical Banach Spaces I: Sequence Spaces, Ergeb. Math. Grenzgeb. 92, Springer, 1977.

[P] A. Pełczyński, Universal bases, Studia Math. 32 (1969), 247-268.

[Ro] H. P. Rosenthal, A characterization of Banach spaces containing $\ell_{1}$, Proc. Nat. Acad. Sci. USA 71 (1974), 2411-2413.

Équipe d'Analyse Fonctionnelle

Université Pierre et Marie Curie - Paris 6

Boîte 186

4 place Jussieu

75252 Paris Cedex 05, France

E-mail:pdodos@math.ntua.gr
Équipe de Logique Mathématiques Université Denis Diderot - Paris 7 2 place Jussieu 72521 Paris Cedex 05, France E-mail: abad@logique.jussieu.fr

Received March 17, 2008

Revised version May 10, 2008 